
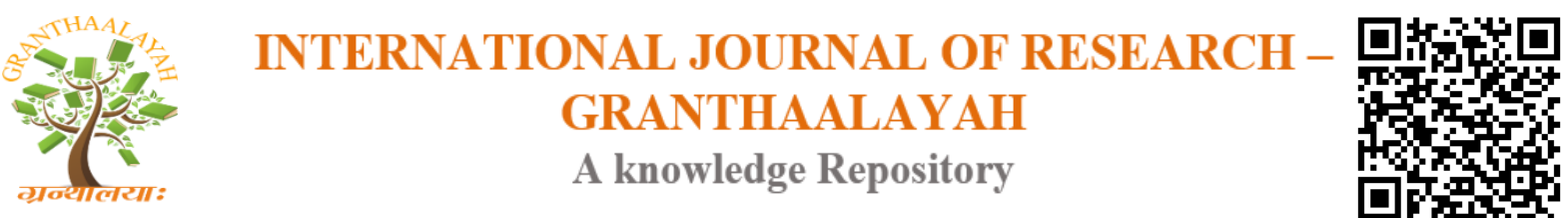

Science

\title{
ON THE POSITIVE PELL EQUATION $Y^{2}=72 X^{2}+36$
}

\author{
M.A.Gopalan ${ }^{1}$, A.Kavitha ${ }^{2}$, A.Jesintha Mary ${ }^{3}$ \\ ${ }^{1}$ Professor, Department of Mathematics, Shrimati Indira Gandhi College, Trichy-620 002, India \\ ${ }^{2}$ Assistant Professor, Department of Mathematics, Shrimati Indira Gandhi College, Trichy-620 \\ 002, India \\ ${ }^{3}$ M.Phil Scholar, Department of Mathematics, Shrimati Indira Gandhi College, Trichy-620 002, \\ India
}

\begin{abstract}
The binary quadratic equation represented by the positive pellian $y^{2}=72 x^{2}+36$ is analysed for its distinct integer solutions. A few interesting relations among the solutions are given. Further, employing the solutions of the above hyperbola, we have obtained solutions of other choices of hyperbolas, parabolas and special Pythagorean triangle.
\end{abstract}

Keywords: Binary Quadratic; Hyperbola; Parabola; Integral Solutions; Pell Equation.

2010 Mathematics Subject Classification: 11D09.

Cite This Article: M.A.Gopalan, A.Kavitha, and A.Jesintha Mary. (2017). "ON THE POSITIVE PELL EQUATION $\mathrm{Y}^{2}=72 \mathrm{X}^{2}+36$." International Journal of Research - Granthaalayah, 5(7:SE), 68-74. https://doi.org/10.29121/granthaalayah.v5.i7(SE).2017.2126.

\section{Introduction}

The binary quadratic equation of the form $y^{2}=D x^{2}+1$ where $\mathrm{D}$ is non-square positive integer has been studied by various mathematicians for its non-trivial integral solutions when $\mathrm{D}$ takes different values [1-4]. For an extensive review of various problems, one may refer [5-20]. In this communication, yet another interesting hyperbola given by $y^{2}=72 x^{2}+36$ is considered and infinitely many integer solutions are obtained. A few interesting properties among the solutions are presented.

\section{Method of Analysis}

Consider the binary quadratic equation

$$
y^{2}=72 x^{2}+36
$$

whose smallest positive integer solution is $x_{0}=2, y_{0}=18$ 
To obtain the other solutions of (1), consider the pell equation $y^{2}=72 x^{2}+1$ whose solution is given by

$$
\tilde{x}_{n}=\frac{1}{2 \sqrt{72}} g_{n}, \tilde{y}_{n}=\frac{1}{2} f_{n}
$$

where,

$$
\begin{aligned}
& f_{n}=(17+2 \sqrt{72})^{n+1}+(17-2 \sqrt{72})^{n+1} \\
& g_{n}=(17+2 \sqrt{72})^{n+1}-(17-2 \sqrt{72})^{n+1}, n=0,1,2,3, \ldots
\end{aligned}
$$

Applying Brahamagupta Lemma between $\left(x_{0}, y_{0}\right)$ and $\left(\tilde{x}_{n}, \tilde{y}_{n}\right)$, the other integer solutions of (1) are given by

$$
\begin{aligned}
& \sqrt{72} x_{n+1}=\sqrt{72} f_{n}+9 g_{n} \\
& y_{n+1}=9 f_{n}+\sqrt{72} g_{n}
\end{aligned}
$$

The recurrence relations satisfied by the solutions (2) are given by

$$
\begin{aligned}
& x_{n+3}-34 x_{n+2}+x_{n+1}=0 \\
& y_{n+3}-34 y_{n+2}+y_{n+1}=0
\end{aligned}
$$

Some numerical examples of $x \& y$ satisfying (1) are given in the Table 1 below:

Table 1: Examples

\begin{tabular}{|l|l|l|}
\hline$n$ & $x_{n}$ & $y_{n}$ \\
\hline 0 & 2 & 18 \\
\hline 1 & 70 & 594 \\
\hline 2 & 2378 & 20178 \\
\hline 3 & 80782 & 685458 \\
\hline 4 & 2744210 & 23285394 \\
\hline
\end{tabular}

From the above table, we observe some interesting relations among the solutions which are presented below:

1) $x_{n}$ and $y_{n}$ values are always even.

2) Each of the following expressions is a nasty number:

- $3 x_{2 n+3}-99 x_{2 n+2}+12$

- $\frac{3 x_{2 n+4}-3363 x_{2 n+2}+408}{34}$

- $6 y_{2 n+3}-1680 x_{2 n+2}+204$

17

- $\frac{6 y_{2 n+4}-57072 x_{2 n+2}+6924}{577}$

- $\frac{198 y_{2 n+2}-48 x_{2 n+3}+204}{17}$

- $198 y_{2 n+3}-1680 x_{2 n+3}+12$ 
- $\frac{198 y_{2 n+4}-57072 x_{2 n+3}+204}{17}$

- $\frac{6726 y_{2 n+2}-48 x_{2 n+4}+6924}{577}$

- $\frac{6726 y_{2 n+3}-1680 x_{2 n+4}+204}{17}$

- $6726 y_{2 n+4}-57072 x_{2 n+4}+12$

- $99 x_{2 n+4}-3363 x_{2 n+3}+12$

- $\frac{35 y_{2 n+2}-y_{2 n+3}+36}{3}$

- $\frac{1189 y_{2 n+2}-y_{2 n+4}+1224}{102}$

- $\frac{1189 y_{2 n+3}-35 y_{2 n+4}+36}{3}$

3) Each of the following expressions is a cubical integer:

- $4\left[x_{3 n+4}-33 x_{3 n+3}+12\left(x_{n+2}-33 x_{n+1}\right)\right]$

- $4624\left[x_{3 n+5}-1121 x_{3 n+3}+3\left(x_{n+3}-1121 x_{n+1}\right)\right]$

- $23409\left[9 y_{3 n+4}-2520 x_{3 n+3}+3\left(9 y_{n+2}-2520 x_{n+1}\right)\right]$

- $26967249\left[9 y_{3 n+5}-85608 x_{3 n+3}+3\left(9 y_{n+3}-85608 x_{n+1}\right)\right]$

- $23409\left[297 y_{3 n+3}-72 x_{3 n+4}+3\left(297 y_{n+1}-72 x_{n+2}\right)\right]$

- $3\left[297 y_{3 n+4}-2520 x_{3 n+4}+3\left(297 y_{n+2}-2520 x_{n+2}\right)\right]$

- $23409\left[297 y_{3 n+5}-85608 x_{3 n+4}+3\left(297 y_{n+3}-85608 x_{n+2}\right)\right]$

- $26967249\left[10089 y_{3 n+3}-72 x_{3 n+5}+3\left(10089 y_{n+1}-72 x_{n+3}\right)\right]$

- $23409\left[10089 y_{3 n+4}-2520 x_{3 n+5}+3\left(10089 y_{n+2}-2520 x_{n+3}\right)\right]$

- $3\left[10089 y_{3 n+5}-85608 x_{3 n+5}+3\left(10089 y_{n+3}-85608 x_{n+3}\right)\right]$

- $324\left[297 x_{3 n+5}-10089 x_{3 n+4}+3\left(297 x_{n+3}-10089 x_{n+2}\right)\right]$

- $324\left[35 y_{3 n+3}-y_{3 n+4}+3\left(35 y_{n+1}-y_{n+2}\right)\right]$

- $374544\left[1189 y_{3 n+3}-y_{3 n+5}+3\left(1189 y_{n+1}-y_{n+3}\right)\right]$

- $324\left[1189 y_{3 n+4}-35 y_{3 n+5}+3\left(1189 y_{n+2}-35 y_{n+3}\right)\right]$

4) Relations among the solutions:

- $18 x_{n+3}=612 x_{n+2}-18 x_{n+1}$

- $18 y_{n+1}=9 x_{n+2}-153 x_{n+1}$

- $18 y_{n+2}=153 x_{n+2}-9 x_{n+1}$

- $18 y_{n+3}=5193 x_{n+2}-153 x_{n+1}$

- $612 y_{n+1}=9 x_{n+3}-5193 x_{n+1}$ 
- $612 y_{n+2}=153 x_{n+3}-153 x_{n+1}$

- $612 y_{n+3}=5193 x_{n+3}-9 x_{n+1}$

- $153 y_{n+1}=9 y_{n+2}-1296 x_{n+1}$

- $5193 y_{n+1}=9 y_{n+3}-44064 x_{n+1}$

- $5193 y_{n+2}=153 y_{n+3}-1296 x_{n+1}$

- $9 y_{n+1}=153 y_{n+2}-1296 x_{n+2}$

- $153 y_{n+1}=153 y_{n+3}-44064 x_{n+2}$

- $153 y_{n+2}=9 y_{n+3}-1296 x_{n+2}$

- $153 y_{n+3}=9 y_{n+2}+1296 x_{n+3}$

- $9 x_{n+2}=-18 y_{n+3}+153 x_{n+3}$

- $9 y_{n+1}=5193 y_{n+3}-44064 x_{n+3}$

- $18 y_{n+1}=153 x_{n+3}-5193 x_{n+2}$

- $18 y_{n+2}=9 x_{n+3}-153 x_{n+2}$

- $1296 x_{n+3}=-153 y_{n+1}+5193 y_{n+2}$

- $18 y_{n+3}=-18 y_{n+1}+612 y_{n+2}$

\section{Remarkable Observation}

I. Employing linear combinations among the solutions of (1), one may generate integer solutions for other choices of hyperbola which are presented in the Table 2 below:

Table 2: Hyperbolas

\begin{tabular}{|l|l|l|}
\hline S.no & \multicolumn{1}{|c|}{$(X, Y)$} & \multicolumn{1}{c|}{ Hyperbola } \\
\hline 1 & $\left(35 x_{n+1}-x_{n+2}, x_{n+2}-33 x_{n+1}\right)$ & $81 Y^{2}-72 X^{2}=1296$ \\
\hline 2 & $\left(1189 x_{n+1}-x_{n+3}, x_{n+3}-1121 x_{n+1}\right)$ & $81 Y^{2}-72 X^{2}=1498176$ \\
\hline 3 & $\left(297 x_{n+1}-y_{n+2}, 9 y_{n+2}-2520 x_{n+1}\right)$ & $Y^{2}-72 X^{2}=93636$ \\
\hline 4 & $\left(10089 x_{n+1}-y_{n+3}, 9 y_{n+3}-85608 x_{n+1}\right)$ & $Y^{2}-72 X^{2}=107868996$ \\
\hline 5 & $\left(9 x_{n+2}-35 y_{n+1}, 297 y_{n+1}-72 x_{n+2}\right)$ & $Y^{2}-72 X^{2}=93636$ \\
\hline 6 & $\left(297 x_{n+2}-35 y_{n+2}, 297 y_{n+2}-2520 x_{n+2}\right)$ & $Y^{2}-72 X^{2}=324$ \\
\hline 7 & $\left(10089 x_{n+2}-35 y_{n+3}, 297 y_{n+3}-85608 x_{n+2}\right)$ & $Y^{2}-72 X^{2}=93636$ \\
\hline 8 & $\left(9 x_{n+3}-1189 y_{n+1}, 10089 y_{n+1}-72 x_{n+3}\right)$ & $Y^{2}-72 X^{2}=107868996$ \\
\hline 9 & $\left(297 x_{n+3}-1189 y_{n+2}, 10089 y_{n+2}-2520 x_{n+3}\right)$ & $Y^{2}-72 X^{2}=93636$ \\
\hline 10 & $\left(10089 x_{n+3}-1189 y_{n+3}, 10089 y_{n+3}-85608 x_{n+3}\right)$ & $Y^{2}-72 X^{2}=324$ \\
\hline 11 & $\left(1189 x_{n+2}-35 x_{n+3}, 297 x_{n+3}-10089 x_{n+2}\right)$ & $Y^{2}-72 X^{2}=1296$ \\
\hline
\end{tabular}




\begin{tabular}{|l|l|l|}
\hline 12 & $\left(y_{n+2}-33 y_{n+1}, 35 y_{n+1}-y_{n+2}\right)$ & $288 Y^{2}-324 X^{2}=373248$ \\
\hline 13 & $\left(y_{n+3}-1121 y_{n+1}, 1189 y_{n+1}-y_{n+3}\right)$ & $2312 Y^{2}-2601 X^{2}=$ \\
& $\left(297 y_{n+3}-10089 y_{n+2}, 1189 y_{n+2}-35 y_{n+3}\right)$ & 7463782912 \\
\hline 14 & $72 Y^{2}-X^{2}=93312$ \\
\hline
\end{tabular}

II. Employing linear combinations among the solutions of (1), one may generate integer solutions for other choices of parabola which are presented in the Table 3 below:

Table 3: Parabolas

\begin{tabular}{|c|c|c|}
\hline S.no & $(X, Y)$ & Parabola \\
\hline 1 & $\left(35 x_{n+1}-x_{n+2}, x_{2 n+3}-33 x_{2 n+2}\right)$ & $72 X^{2}=162 Y-648$ \\
\hline 2 & $\left(1189 x_{n+1}-x_{n+3}, x_{2 n+4}-1121 x_{2 n+2}\right)$ & $72 X^{2}=5508 Y-749088$ \\
\hline 3 & $\left(297 x_{n+1}-y_{n+2}, 9 y_{2 n+3}-2520 x_{2 n+2}\right)$ & $72 X^{2}=153 Y-46818$ \\
\hline 4 & $\left(10089 x_{n+1}-y_{n+3}, 9 y_{2 n+4}-85608 x_{2 n+2}\right)$ & $72 X^{2}=5193 Y-53934498$ \\
\hline 5 & $\left(9 x_{n+2}-35 y_{n+1}, 297 y_{2 n+2}-72 x_{2 n+3}\right)$ & $72 X^{2}=153 Y-46818$ \\
\hline 6 & $\left(297 x_{n+2}-35 y_{n+2}, 297 y_{2 n+3}-2520 x_{2 n+3}\right)$ & $72 X^{2}=9 Y-162$ \\
\hline 7 & $\left(10089 x_{n+2}-35 y_{n+3}, 297 y_{2 n+4}-85608 x_{2 n+3}\right)$ & $72 X^{2}=153 Y-46818$ \\
\hline 8 & $\left(9 x_{n+3}-1189 y_{n+1}, 10089 y_{2 n+2}-72 x_{2 n+4}\right)$ & $72 X^{2}=5193 Y-539344$ \\
\hline 9 & $\left(297 x_{n+3}-1189 y_{n+2}, 10089 y_{2 n+3}-2520 x_{2 n+4}\right)$ & $72 X^{2}=153 Y-46818$ \\
\hline 10 & $\left(\begin{array}{l}10089 x_{n+3}-1189 y_{n+3}, \\
10089 y_{2 n+4}-85608 x_{2 n+4}\end{array}\right)$ & $72 X^{2}=9 Y-162$ \\
\hline 11 & $\left(1189 x_{n+2}-35 x_{n+3}, 297 x_{2 n+4}-10089 x_{2 n+3}\right)$ & $72 X^{2}=18 Y-648$ \\
\hline 12 & $\left(y_{n+2}-33 y_{n+1}, 35 y_{2 n+2}-y_{2 n+3}\right)$ & $X^{2}=16 Y-576$ \\
\hline 13 & $\left(y_{n+3}-1121 y_{n+1}, 1189 y_{2 n+2}-y_{2 n+4}\right)$ & $X^{2}=544 Y-665856$ \\
\hline 14 & $\left(297 y_{n+3}-10089 y_{n+2}, 1189 y_{2 n+3}-35 y_{2 n+4}\right)$ & $X^{2}=1296 Y-46656$ \\
\hline
\end{tabular}

III. Consider $m=x_{n+1}+y_{n+1}, n=x_{n+1}$. Observe that $m>n>0$. Treat $\mathrm{m}, \mathrm{n}$ as the generators of the Pythagorean Triangle $T(\alpha, \beta, \gamma)$, where $\alpha=2 m n, \beta=m^{2}-n^{2}, \gamma=m^{2}+n^{2}$

Then the following interesting relations are observed:

a) $\alpha-36 \beta+35 \gamma=-36$

b) $37 \alpha-\gamma+\frac{144 A}{P}=36$

c) $-34 \gamma+36 \beta-38 \alpha+\frac{144 A}{P}=72$ 


$$
\text { d) } \frac{2 A}{P}=x_{n+1} y_{n+1}
$$

\section{Conclusion}

In this paper, we have presented infinitely many integer solutions for the hyperbola represented by the positive Pell equation $y^{2}=72 x^{2}+36$. As the binary quadratic Diophantine equations are rich in variety, one may search for the other choices of positive Pell equations and determine their integer solutions along with suitable properties.

\section{References}

[1] Dickson L.E., "History of theory of Number", Chelsa Publishing Company, (vol-2), Newyork, 1952.

[2] Mordel L.J ., "Diophantine Equations", Academic Press, New york, 1969.

[3] Telang S.J., "Number theory", Tata McGraw Hill Publishing Company Limited, New Delhi, 2000.

[4] David M.Burton., "Elementary Number Theory", Tata McGraw Hill Publishing Company Limited, New Delhi, 2002.

[5] Gopalan M.A., Vidhyalakshmi $S$ and Devibala $S$, "On the Diophantine Equation $3 x^{2}+x y=14$, Acta Cinecia Indica, 2007, vol-XXXIIIM, No.2, 645-648.

[6] Gopalan M.A and Janaki G, "Observations on $y^{2}=3 x^{2}+1$ ", Acta Cinecia Indica, vol-XXIVM, 2008, No.2, 693-696.

[7] Gopalan M.A and Sangeetha G, "A Remarkable Observation on $y^{2}=10 x^{2}+1$ ", Impact Journal of science and Technology, 2010, 4(1), 103-106.

[8] Gopalan M.A and Vijayalakshmi R, "Observations on the integral solutions of $y^{2}=5 x^{2}+1$, Impact Journal of science and Technology, 2010, 4(4), 125-129.

[9] Gopalan M.A and Sivakami B, "Observations on the integral solutions of $y^{2}=7 x^{2}+1$ ", Antarctica Journal of Mathematics, 2010, 7(3), 291-296.

[10] Gopalan M.A and Yamuna R.S, "Remarkable Observations on the binary quadratic equation $y^{2}=\left(k^{2}+1\right) x^{2}+1, k \in z-\{0\}$, Impact Journal of science and Technology, 2010, 4(4), 61-65.

[11] Gopalan M.A and Vijayalakshmi R, "Special Pythagorean triangle generated through the integral solutions of the equation $y^{2}=\left(k^{2}-1\right) x^{2}+1$, Antarctica Journal of Mathematics, 2010, 7(5), 503-507.

[12] Gopalan M.A and Srividhya G, "Relations among M-gonal Number through the equation $y^{2}=2 x^{2}+1$, , Antarctica Journal of Mathematics, 2010, 7(3), 363-369.

[13] Gopalan M.A and Palanikumar R, "Observation on $y^{2}=12 x^{2}+1$ ", Antarctica Journal of Mathematics, 2011, 8(2), 149-152.

[14] Gopalan M.A., Vidhyalakshmi S., Usha Rani T.R and Mallika S, "Observations on $y^{2}=12 x^{2}-3$,, Bessel Journal of Math, 2012 ,2(3), 153-158.

[15] Gopalan M.A., Vidhyalakshmi S., Umarani J, "Remarkable Observations on the hyperbola $y^{2}=24 x^{2}+1$,, Bulletin of Mathematics and statistics Research, 2013, 1, 9-12. 
[16] Gopalan M.A., Vidhyalakshmi S., Maheswari D, "Observations on the hyperbola $y^{2}=30 x^{2}+1$,, International Journal of Engineering Research, 2013, 1(3), 312-314.

[17] Geetha T., Gopalan M.A., Vidhyalakshmi S, "Observations on the hyperbola $y^{2}=72 x^{2}+1$ ", Scholars Journal of physics, Mathematics and statistics, 2014, 1(1), 1-3.

[18] Gopalan M.A and Sivakami B, "Special Pythagorean triangle generated through the integral solutions of the equation $y^{2}=\left(k^{2}+2 k\right) x^{2}+1$, , Diaphantus. J.math., 2(1), 2013, 25-30.

[19] Gopalan M.A., Vidhyalakshmi S., Usha Rani T.R., Agalya K, "Observations on the hyperbola $y^{2}=110 x^{2}+1$, , International Journal of multidisciplinary Research and Development, 2015, vol 2, issue 3, 237-239.

[20] Vidhyalakshmi S., Gopalan M.A., Sumithra S., Thiruniraiselvi N, "Observations on the hyperbola $y^{2}=60 x^{2}+4,$, JIRT, 2014, vol 1, issue 11, 119-121. 